\title{
Quadratic minima and modular forms II
}

\author{
by \\ BARry Brent (Minneapolis, MN)
}

1. Introduction. Carl Ludwig Siegel showed in [S1] (English translation, [S2]) that the constant terms of certain level one negative-weight modular forms $T_{h}$ are non-vanishing (Satz 2), and that this implies an upper bound on the least positive exponent of a non-zero Fourier coefficient for any level one entire modular form of weight $h$ with a non-zero constant term. Level one theta functions fall into this category. Their Fourier coefficients code up representation numbers of quadratic forms. For positive even $h$, Siegel's result gives an upper bound on the least positive integer represented by a positive-definite even unimodular quadratic form in $n=2 h$ variables. This bound is sharper than Minkowski's for large $n$. (Mallows, Odlyzko and Sloane have improved Siegel's bound in [MOS].)

John Hsia [private communication to Glenn Stevens] suggested that Siegel's approach might be extended to higher levels. Following this hint, we constructed an analogue of $T_{h}$ for $\Gamma_{0}(2)$, which we denote as $T_{2, h}$. To prove Satz 2, Siegel controlled the sign of the Fourier coefficients in the principal part of $T_{h}$. In [B] (henceforth, "part I"), following Siegel, we found upper bounds for the first positive exponent of a non-zero Fourier coefficient occurring in the expansion at infinity of an entire modular form with a non-zero constant term for $\Gamma_{0}(2)$ in the case $h \equiv 0(\bmod 4)$. Siegel's method carried over intact.

In part I, we also stated that it was not clear that Siegel's method forces the non-vanishing of the $T_{2, h}$ constant terms when $h \equiv 2(\bmod 4)$. But it turns out that we can tweak our definition of the $T_{2, h}$ and carry out Siegel's strategy.

Let us denote the vector space of entire modular forms of weight $h$ for $\Gamma_{0}(N)$ as $M(N, h)$. In part I, we proved that the second non-zero Fourier coefficient of an element of $M(2, h)$ with non-zero constant term must have exponent at most $\operatorname{dim} M(2, h)=1+\lfloor h / 4\rfloor=r$ (say) if $h \equiv 0(\bmod 4)$. This

2000 Mathematics Subject Classification: 11F11, 11E20. 
corresponds exactly to Siegel's bound for $f \in M(1, h)$. If $h \equiv 2(\bmod 4)$, however, we only showed that the exponent is no more than $2 r$.

In the second section, we prove that the exponent is at most $r$ if $h \equiv 2$ $(\bmod 4)$. In the third section, we apply this result to the theory of quadratic forms.

We show that, if $Q$ is an even positive-definite level two quadratic form in $v=8 u+4$ variables, then $Q$ represents a positive integer $2 n \leq 1+v / 4$. (In part I, we obtained the weaker bound $2+v / 2$. We also showed that if $v=8 u$, then $Q$ represents an even positive integer $\leq 2+v / 4$.)

\section{Bounds for gaps in the Fourier expansions of entire modular} forms. Section 2.1 is introductory. All but one of the results are stated without proof. The reader is referred to part I for details. In Section 2.2, we estimate the first positive exponent of a non-zero Fourier coefficient in the expansion of an entire modular form for $\Gamma_{0}(2)$ with a non-zero constant term.

2.1. Some modular objects. This section is a tour of the objects mentioned in the article. The main building blocks are Eisenstein series with known divisors and computable Fourier expansions.

As usual, we denote by $\Gamma_{0}(N)$ the congruence subgroup

$$
\Gamma_{0}(N)=\left\{\left(\begin{array}{ll}
a & b \\
c & d
\end{array}\right) \in \mathrm{SL}(2, \mathbb{Z}): c \equiv 0(\bmod N)\right\} .
$$

The vector space of entire modular forms of one variable in the upper half plane $\mathfrak{H}$ of weight $h$ for $\Gamma_{0}(N)$ ("level $N$ ") and trivial character is denoted by $M(N, h)$. We have an inclusion lattice satisfying:

$$
M(L, h) \subset M(N, h) \text { if and only if } L \mid N .
$$

More particularly, any entire modular form for $\operatorname{SL}(2, \mathbb{Z})$ is also one for $\Gamma_{0}(2)$.

The dimension of $M(N, h)$ is denoted by $r(N, h)$, or $r_{h}$, or by $r$. For any positive even $h$,

$$
r(2, h)=\left\lfloor\frac{h}{4}\right\rfloor+1 .
$$

We write $\Delta$ for the weight 12 , level one cusp form with Fourier series

$$
\Delta=\sum_{n=1}^{\infty} \tau(n) q^{n}
$$

and product expansion

$$
\Delta=q \prod_{n=1}^{\infty}\left(1-q^{n}\right)^{24} .
$$

Here, $\tau$ is the Ramanujan function. 
We describe some level two objects, using three special divisor sums:

and

$$
\sigma^{\text {odd }}(n)=\sum_{\substack{0<d \mid n \\ d \text { odd }}} d, \quad \sigma_{k}^{\text {alt }}(n)=\sum_{0<d \mid n}(-1)^{d} d^{k}
$$

$$
\sigma_{N, k}^{*}(n)=\sum_{\substack{0<d \mid n \\ N \nmid n / d}} d^{k}
$$

Let $E_{\gamma, 2}$ denote the unique normalized form in the one-dimensional space $M(2,2)$ (i.e. the leading coefficient in the Fourier expansion of the form is a 1 ). The Fourier series is

$$
E_{\gamma, 2}=1+24 \sum_{n=1}^{\infty} \sigma^{\text {odd }}(n) q^{n} .
$$

$E_{\gamma, 2}$ has a $\frac{1}{2}$-order zero at points of $\mathfrak{H}$ which are $\Gamma_{0}(2)$-equivalent to $-\frac{1}{2}+$ $\frac{1}{2} i=\gamma$ (say). The vector space $M(2,4)$ is spanned by two forms $E_{0,4}$ and $E_{\infty, 4}$, which vanish with order one at the $\Gamma_{0}(2)$-inequivalent zero and infinity cusps, respectively. They have Fourier expansions

$$
E_{0,4}=1+16 \sum_{n=1}^{\infty} \sigma_{3}^{\text {alt }}(n) q^{n}
$$

and

$$
E_{\infty, 4}=\sum_{n=1}^{\infty} \sigma_{2,3}^{*}(n) q^{n} .
$$

The following lemma was Proposition 2.3 in part I:

LEMma 2.1. The modular form $E_{\infty, 4} \in M(2,4)$ has the following product decomposition in the variable q:

$$
E_{\infty, 4}(z)=q \prod_{0<n \in 2 \mathbb{Z}}\left(1-q^{n}\right)^{8} \prod_{0<n \in \mathbb{Z} \backslash 2 \mathbb{Z}}\left(1-q^{n}\right)^{-8} .
$$

We do not need it, but it is also easy to show that

$$
E_{0,4}(z)=q \prod_{0<n \in 2 \mathbb{Z}}\left(1-q^{n}\right)^{8} \prod_{0<n \in \mathbb{Z} \backslash 2 \mathbb{Z}}\left(1-q^{n}\right)^{16} .
$$

To exploit Lemma 2.1, we need a result due essentially to Euler (Lemma 2.11 of part I, quoted from [A], Theorem 14.8, valid when the infinite series is absolutely convergent):

Lemma 2.2. For a given set $A$ and a given arithmetical function $f$, the numbers $p_{A, f}(n)$ defined by the equation

$$
\prod_{n \in A}\left(1-x^{n}\right)^{-f(n) / n}=1+\sum_{n=1}^{\infty} p_{A, f}(n) x^{n}
$$


satisfy the recursion formula

where $p_{A, f}(0)=1$ and

$$
n p_{A, f}(n)=\sum_{k=1}^{n} f_{A}(k) p_{A, f}(n-k),
$$

$$
f_{A}(k)=\sum_{\substack{d \mid k \\ d \in A}} f(d) .
$$

Next, we construct a level two analogue of the level one Klein invariant $j$ :

$$
j_{2}=E_{\gamma, 2}^{2} E_{\infty, 4}^{-1} .
$$

The function $j_{2}$ is analogous to $j$ because it is modular (weight zero) for $\Gamma_{0}(2)$, holomorphic on the upper half plane, has a simple pole at infinity, generates the field of $\Gamma_{0}(2)$-modular functions, and defines a bijection of a $\Gamma_{0}(2)$ fundamental set with $\mathbb{C}$.

The following lemma was Proposition 2.7 in part I:

LEMMA 2.3. For $z \in \mathfrak{H}$,

$$
\frac{d}{d z} j_{2}(z)=-2 \pi i E_{\gamma, 2}(z) E_{0,4}(z) E_{\infty, 4}(z)^{-1}
$$

We introduce a level two analogue of Siegel's $T_{h}$ for $h \equiv 2(\bmod 4)$. For $r=r(2, h)$, we set

$$
t_{h}=E_{0,4} E_{\infty, 4}^{-r} .
$$

The $t_{h}$ are a useful replacement for the functions

$$
T_{2, h}=E_{\gamma, 2}^{2} E_{0,4} E_{\infty, 4}^{-1-r}
$$

we defined in part I for the same $h$.

Finally, for $h \equiv 2(\bmod 4)$ and $f \in M(2, h)$, let

$$
w_{2}(f)=E_{\gamma, 2}^{-1} E_{\infty, 4}^{1-r} f .
$$

This replaces the part I function $W_{2}(f)=E_{\gamma, 2} E_{\infty, 4}^{-(h+2) / 4} f$ defined on the same $f$.

2.2. A structural result on the $q$ series of entire level two modular forms. We establish a sequence of propositions mimicking the argument of [S2], pp. 249-254. (Siegel's proof is also sketched on p. 263 of part I.)

Proposition 2.1. The map $w_{2}$ is a vector space isomorphism from $M(2, h)$ onto the space of polynomials in $j_{2}$ of degree less than $r$.

Pr o of. No non-trivial polynomial in $j_{2}$ can vanish almost everywhere, so the modular forms $j_{2}^{d} E_{\gamma, 2} E_{\infty, 4}^{r-1}, d=0,1, \ldots, r-1$, are a basis for $M(2, h)$, and we have

$$
w_{2}\left(j_{2}^{d} E_{\gamma, 2} E_{\infty, 4}^{r-1}\right)=j_{2}^{d} .
$$

The map $w_{2}$ is clearly linear and 1-to- 1 . 
Proposition 2.2. For $f \in M(2, h)$, the constant term in the Fourier expansion at infinity of $t_{h} f$ is zero.

Pr o of. By applying Lemma 2.3, we see that

$$
w_{2}(f) \frac{d}{d z} j_{2}=-2 \pi i t_{h} f
$$

Thus, $t_{h} f$ is the derivative of a polynomial in $j_{2}$, so it can be expressed in a neighborhood of infinity as the derivative with respect to $z$ of a power series in the variable $q=\exp (2 \pi i z)$. This derivative is a power series in $q$ with vanishing constant term.

Proposition 2.3. For $h \equiv 2(\bmod 4)$, the constant term in the Fourier expansion at infinity of $t_{h}$ is non-zero.

Proof. Lemmas 2.1 and 2.3 imply that, for fixed $s$,

$$
E_{\infty, 4}^{-s}=q^{-s} \sum_{n=0}^{\infty} R(n) q^{n},
$$

where $R(0)=1$ and $n>0$ implies that

$$
R(n)=\frac{8 s}{n} \sum_{a=1}^{n} \sigma_{1}^{\text {alt }}(a) R(n-a) .
$$

The divisor functions $\sigma_{k}^{\text {alt }}(n), k$ odd, alternate sign, so the alternation of the sign of $R(n)$ follows by an easy induction argument from (2.5). To be specific, $R(n)=U_{n}(-1)^{n}$ for some $U_{n}>0$. Thus we may write $E_{\infty, 4}^{-r}=U_{0}(-1)^{0} q^{-r}+U_{1}(-1)^{1} q^{1-r}+\ldots+U_{r-1}(-1)^{r-1} q^{-1}+U_{r}(-1)^{r}+\ldots$

On the other hand, the Fourier coefficient of $q^{n}, n \geq 0$, in the expansion of $E_{0,4}$ is $W_{n}(-1)^{n}$ for positive $W_{n}$, by (2.2). Thus the constant term of $t_{h}=E_{0,4} E_{\infty, 4}^{-r}$ is

$$
\sum_{m=-r}^{0} U_{m}(-1)^{m} W_{r-m}(-1)^{r-m} \neq 0 .
$$

What follows is our main theorem on modular forms.

TheOREM 2.1. Suppose $f \in M(2, h)$ with Fourier expansion at infinity

$$
f(z)=\sum_{n=0}^{\infty} A_{n} q^{n}, \quad A_{0} \neq 0 .
$$

Then some $A_{n} \neq 0,1 \leq n \leq r(2, h)$.

Proof. First suppose that $h \equiv 2(\bmod 4)$. We denote the coefficient of $q^{n}$ in the Fourier expansion of $f$ at infinity as $c_{n}[f]$. The normalized 
meromorphic form $t_{h}$ has a Fourier series of the form

$$
t_{h}=C_{h,-r} q^{-r}+\ldots+C_{h, 0}+\ldots,
$$

with $C_{h,-r}=1$. By Proposition 2.2,

$$
0=c_{0}\left[t_{h} f\right]=C_{h, 0} A_{0}+\ldots+C_{h,-r} A_{r} .
$$

By hypothesis, $A_{0} \neq 0$. By Proposition 2.3, $C_{h, 0} \neq 0$, so

$$
A_{0}=-\left(C_{h, 0}\right)^{-1}\left(C_{h,-1} A_{1}+\ldots+C_{h,-r} A_{r}\right) .
$$

It follows that one of the $A_{n}(n=1, \ldots, r)$ is non-zero.

To complete the proof, we point out that the claim was proved for $h \equiv 0$ $(\bmod 4)$ in Theorem 2.12 of part I by the same sort of argument.

REMARK This result is slightly better than the bound $n \leq r+1$ of Conjecture 6.2 , part I for $h \equiv 2(\bmod 4)$. The reason is our different choice of a level two $T_{h}$ analogue.

\section{Quadratic minima}

3.1. Quadratic forms and modular forms. For even $v$, we write $\mathbf{x}=$ ${ }^{t}\left(x_{1}, \ldots, x_{v}\right)$, so that $\mathbf{x}$ is a column vector. Let $A$ be an $v$ by $v$ square symmetric matrix with integer entries, even entries on the diagonal, and positive eigenvalues. Then $Q_{A}(\mathbf{x})={ }^{t} \mathbf{x} A \mathbf{x}$ is a homogeneous second degree polynomial in the $x_{i}$. We refer to $Q_{A}$ as the even positive-definite quadratic form associated with $A$. If $\mathbf{x} \in \mathbb{Z}^{v}$, then $Q_{A}(\mathbf{x})$ is a non-negative even number, which is zero only if $\mathbf{x}$ is the zero vector. The level of $Q_{A}$ is the smallest positive integer $N$ such that $N A^{-1}$ also has integer entries and even entries on the diagonal. Let $\# Q_{A}^{-1}(n)$ denote the cardinality of the inverse image in $\mathbb{Z}^{v}$ of an integer $n$ under the quadratic form $Q_{A}$.

The following specialization of known results was Proposition 5.1 of part I.

Lemma 3.1. Suppose that $Q_{A}$ is a level two quadratic form. Then the function $\Theta_{A}: \mathfrak{H} \rightarrow \mathbb{C}$ satisfying

$$
\Theta_{A}(z)=\sum_{n=0}^{\infty} \# Q_{A}^{-1}(2 n) q^{n}
$$

lies in $M(2, v / 2)$.

Since $M(2, h)$ is non-trivial only for even $h$, it also follows that $4 \mid v$.

3.2. Quadratic minima. In this section we apply Theorem 2.1 to the problem of quadratic minima.

TheOREM 3.1. If $Q$ is a level two even positive-definite quadratic form in $v$ variables, $8 \mid v$, then $Q$ represents a positive integer $2 n \leq 2+v / 4$. If $v \equiv 4(\bmod 8)$, then $Q$ represents a positive integer $2 n \leq 1+v / 4$. 
Proof. The claim for $8 \mid v$ was included in Theorem 5.2 of part I. Suppose $v=8 u+4$. Let $A$ be the matrix associated with $Q$, so that $Q=Q_{A}$. Then $\Theta_{A} \in M(2,4 u+2)$, and $\# Q_{A}^{-1}(2 n) \neq 0$ for some $n, 1 \leq n \leq$ $r(2,4 u+2)=1+u$. Thus $Q$ represents an integer $2 n \leq 2+2 u=1+v / 4$.

\section{References}

[A] T. M. Apostol, Introduction to Analytic Number Theory, Springer, New York, 1976.

[B] B. Brent, Quadratic minima and modular forms, Experiment. Math. 7 (1998), 257-274.

[MOS] C. L. Mallows, A. M. Odlyzko and N. J. A. Sloane, Upper bounds for modular forms, lattices, and codes, J. Algebra 36 (1975), 68-76.

[S1] C. L. Siegel, Berechnung von Zetafunktionen an ganzzahligen Stellen, Nachr. Akad. Wiss. Göttingen Math. 10 (1969), 87-102.

[S2] —, Evaluation of zeta functions for integral values of arguments, in: Advanced Analytic Number Theory, Tata Institute of Fundamental Research, Bombay, 1980, 249-268.

Department of Mathematics

University of Minnesota

Vincent Hall

206 Church Street S.E.

Minneapolis, MN 55455, U.S.A.

E-mail: barryb@primenet.com 